\title{
Aliskiren prevents and ameliorates metabolic syndrome in fructose-fed rats
}

Chu-Lin Chou ${ }^{1,2}$, Yu-Hsien Lai ${ }^{3}$, Teng-Yi Lin ${ }^{4}$, Tony J.F. Lee ${ }^{5,6}$, Te-Chao Fang ${ }^{1,3,7}$

\author{
${ }^{1}$ Institute of Medical Sciences, Medical College, Tzu-Chi University, Hualien, Taiwan \\ 2Department of Medicine, Hualien Armed Forces General Hospital, Hualien, Taiwan \\ ${ }^{3}$ Division of Nephrology, Buddhist Tzu Chi General Hospital, Hualien, Taiwan \\ ${ }^{4}$ Department of Laboratory Medicine, Buddhist Tzu Chi General Hospital, Hualien, \\ Taiwan \\ IInstitutes of Life Sciences, Pharmacology and Toxicology, and Medical Sciences, \\ Tzu-Chi University, Hualien, Taiwan \\ 6Department of Pharmacology, Southern Illinois University School of Medicine, \\ Springfield, IL, USA \\ 7Department of Medicine, Medical College, Tzu Chi University, Hualien, Taiwan
}

Submitted: 8 November 2010

Accepted: 7 December 2010

Arch Med Sci 2011; 7, 5: 882-888

DOI: 10.5114/aoms.2011.25566

Copyright (c) 2011 Termedia \& Banach

\section{Abstract}

Introduction: The renin-angiotensin system plays a major role in the pathogenesis of metabolic syndrome. The objective of this study was to examine the effects of aliskiren, a direct renin inhibitor, on the metabolic syndrome of fructose-fed rats.

Material and methods: Male Sprague-Dawley rats were divided into 4 groups ( $n=6$ for each group). Group Con: rats were fed a standard chow diet for 8 weeks, group Fru: rats were fed a high fructose diet (60\% fructose) for 8 weeks, group FruA: rats were fed a high fructose diet and were co-infused with aliskiren $(100 \mathrm{mg} / \mathrm{kg} / \mathrm{day})$, and group FruB: rats were treated as group Fru, but aliskiren was administered 4 weeks later. Systolic blood pressure (SBP), homeostasis model assessment-insulin resistance (HOMA-IR), and blood profiles were measured.

Results: By the end of week 4 and 8 of a high fructose diet, SBP had increased significantly from $111 \pm 5$ to $142 \pm 4$ and $139 \pm 5 \mathrm{mmHg}(p<0.05)$, respectively. A high fructose diet significantly increased HOMA-IR from baseline $(6.15 \pm 1.59)$ to $21.25 \pm 2.08$ and $21.28 \pm 3.1(p<0.05)$ at week 4 and 8 , respectively, and significantly induced metabolic syndrome. Concurrent aliskiren treatment prevented the development of hypertension and metabolic syndrome in fructosefed rats. When fructose-induced hypertension was established, subsequent aliskiren treatment for 4 weeks reversed the elevated SBP and ameliorated metabolic syndrome. There were no significant differences in food, water intake, urine flow or body weight gain among groups.

Conclusions: Aliskiren not only prevents but also ameliorates metabolic syndrome in fructose-fed rats.

Key words: aliskiren, direct renin inhibitor, fructose, hypertension, metabolic syndrome.

\section{Introduction}

Metabolic syndrome, a worldwide issue, has been regarded as the existing development of overall and central obesity, impaired glucose tolerance, dyslipidaemia (combination of low levels of high-density
Corresponding author:

Te-Chao Fang MD, PhD Division of Nephrology Buddhist Tzu Chi General Hospital 707, Section 3 Chung Yang Rd Hualien 97004, Taiwan Phone: +886-3-856-1825, ext. 2253

Fax: +886-3-856-4673

E-mail:

fangtechao@yahoo.com.tw 
lipoprotein cholesterol and high levels of triglycerides), and hypertension [1, 2]. Similarly, emerging data suggest that experimental fructose consumption tends to produce some of the changes associated with metabolic syndrome even without increasing body weight [3]. The detrimental metabolic processes related to fructose consumption seem unlimited to obesity-pathway mechanisms [3]. Therefore, this animal model of fructose-fed rats is popularly utilized to study the metabolic disturbances and hypertension, independent of obesity or genetic contributions.

It is well recognized that the renin-angiotensin system (RAS) plays an important physiological role in the control of body fluid and blood pressure homeostasis, through its effects on cardiovascular, renal and neural functions [4]. Our previous studies demonstrated that angiotensin (Ang) converting enzyme (ACE) inhibitor and Ang II type 1 receptor blockers (ARB) reduce blood pressure and improve insulin sensitivity in hyperinsulinaemia-induced hypertensive rats $[5,6]$. These results are consistent with reports by others [7, 8]. For example, recent clinical trials demonstrated that the use of an ACE inhibitor and ARB significantly reduced the risk of developing diabetes compared with other classes of antihypertensive agents [7, 8], indicating that inhibition of the RAS may contribute to the improvement of insulin resistance. Similarly, Aliskiren, a renin inhibitor, has been demonstrated to reduce blood pressure in spontaneously hypertensive rats [9], essential hypertensive patients and type 2 diabetes with hypertension $[10,11]$. Further, the effects of aliskiren on the metabolic syndrome are currently under investigation. Therefore, we undertook this study to evaluate the effects of aliskiren on the metabolic syndrome in rats fed with a high fructose diet.

\section{Material and methods}

\section{Animals}

Male Sprague-Dawley rats initially weighing $200 \mathrm{~g}$ to $230 \mathrm{~g}$ were used for the experiments. All experimental procedures were carried out in accordance with prior approval of the Institutional Animal Care and Use Committee of Tzu Chi University (TCU 98-05). Rats were kept in a room with air maintained at a temperature of $24-27^{\circ} \mathrm{C}$, humidity of $50-80 \%$ and a 12 -h light/dark cycle, and had access to tap water ad libitum throughout the experiments.

The standard chow diet was composed of $50 \%$ starch, $21 \%$ protein, $4 \%$ fat, $4.5 \%$ cellulose, and standard vitamins and mineral mix. The high fructose diet (Harlan Teklad, Madison, WI) was composed of $60 \%$ fructose, $21 \%$ protein, $5 \%$ fat, $8 \%$ cellulose, and standard vitamins and mineral mix.

\section{Experimental protocols}

After a control period of 1 week, rats were divided into 4 groups ( $n=6$ for each group). Group Con: rats were fed a standard chow diet for 8 weeks and served as the control group, group Fru: rats were fed a high fructose diet for 8 weeks, group FruA: rats were fed a high fructose diet and were co-infused with aliskiren (100 mg/kg per day, a kind gift of Novartis Pharmaceuticals, Basel, Switzerland) via a subcutaneous osmotic minipump, and group FruB: rats were treated as group Fru, but aliskiren (100 mg/kg per day) was administered 4 weeks later. The first day of fructose feeding was recognized as day 1 .

Body weight was measured twice a week. The systolic blood pressure (SBP) was measured twice a week by the tail-cuff method. Blood samples (1 $\mathrm{ml})$ for glucose, insulin, triglycerides, and total cholesterol were taken after $12 \mathrm{~h}$ of fasting at day 0 , midpoint (day 28), and at the end of the study (day 56) from the femoral artery under sodium pentobarbital anaesthesia (40 mg/kg, IP). Plasma was separated, divided into aliquots, and frozen until analysis.

In order to assess insulin resistance of animals, homeostasis model assessment-insulin resistance (HOMA-IR) was determined at day 0 , day 28 , and day 56 of fructose treatment.

\section{Measurements for systolic blood pressure}

Rats were removed from the animal room and were taken to the laboratory at 8 AM; they were allowed free access to water and were kept in a quiet area before SBP was measured at 9 AM. The tail-cuff method without heating was used to measure SBP with the use of a programmed electrosphygmomanometer (MK-2000ST, Muromachi, Tokyo, Japan) as described previously $[5,6]$. The mean of 6 consecutive readings was used as the measurement of SBP of each rat for that day, and SBP was determined twice a week during the control (1 week) and experimental (8 weeks) periods.

\section{Osmotic minipump installation}

The osmotic minipump installation was done as in our previous studies $[5,6]$. In brief, when aliskiren was administered to rats, an osmotic minipump (No. 2002, 14 days of active life, Alza Corp) was filled with the agent, which was dissolved in normal saline, and was implanted subcutaneously in rats under brief anaesthesia with ketamine $(60 \mathrm{mg} / \mathrm{kg}$, IP) and xylazine $(7.5 \mathrm{mg} / \mathrm{kg}$, IP). Aqueous penicillin (5000 U/kg SC) was administered immediately after minipump implantation. At the end of the life of the minipump, a new minipump was implanted and the used one removed. The residual volume in each 


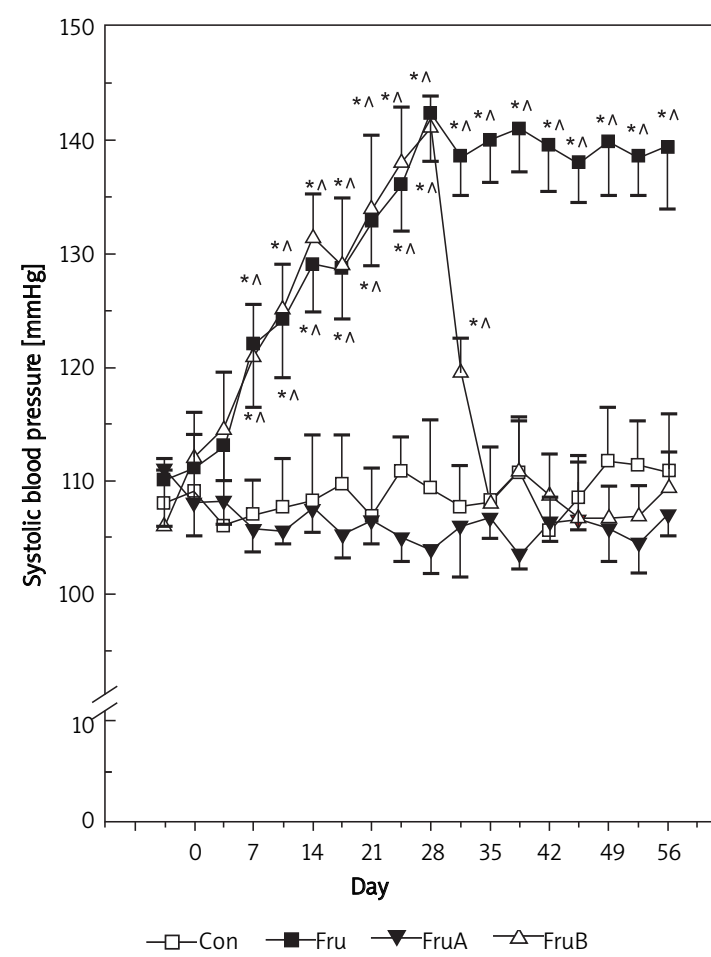

Figure 1. Changes in systolic blood pressure in control and fructose-fed rats with or without direct renin inhibitor (aliskiren) treatment

Group Con - rats were fed standard chow diet, group Fru rats were fed high fructose diet, group FruA - rats were fed high fructose diet and were co-infused with aliskiren, group FruB - rats were treated as group Fru, but aliskiren was administered 4 weeks later, ${ }^{*}$ and $\wedge$ denote $p<0.05$ vs. prefructose and control rats, respectively, $n=6$ for each group

minipump removed was carefully examined to ensure that the minipump release function was normal.

\section{Homeostatic model assessment-insulin resistance (HOMA-IR)}

Insulin resistance was assessed via HOMA-IR, and was determined at day 0 , day 28, and day 56 . HOMA-IR was calculated using this formula: [plasma glucose $(\mathrm{mmol} / \mathrm{l}) \times$ insulin $(\mathrm{mU} / \mathrm{ml})] / 22.5$.

\section{Laboratory measurements}

The blood samples were immediately centrifuged at $4000 \mathrm{~g}$ at $4^{\circ} \mathrm{C}$ for $10 \mathrm{~min}$. The plasma samples were separated and subjected to immediate assays of glucose, insulin, triglycerides and total cholesterol. Plasma levels of glucose, triglycerides (TG) and total cholesterol were measured with a COBAS Integra 800 analyser (Roche Diagnostics, USA). Plasma insulin was measured by automated chemiluminescence immunoassay via an ADVIA Centaur analyser (Bayer, Germany).

\section{Statistical analysis}

All results were presented as mean $\pm \mathrm{SD}$. Experimental data over time were analysed by two-way analysis of variance (ANOVA) (the first factor being the treatment group and the second the time period) for comparison between groups. When a significant effect was detected by ANOVA, the Newman-Keuls test was used to establish which difference between means reached statistical significance. Student's $t$-test for unpaired data was also performed when appropriate. Value of $p<0.05$ was considered statistically significant.

\section{Results}

Aliskiren prevents and reduces the rise of SBP in fructose-fed rats

The changes in SBP of control rats and fructosefed rats with or without aliskiren treatment are illustrated in Figure 1. In control rats, SBP did not change significantly throughout the experimental period. However, high fructose diet alone significantly increased the SBP from $111 \pm 5 \mathrm{mmHg}$ during the control period to $142 \pm 4$ and $139 \pm 5 \mathrm{mmHg}$ by the end of the $4^{\text {th }}$ and $8^{\text {th }}$ weeks, respectively. Concomitant aliskiren treatment prevented the rise of SBP in fructose-fed rats, and the levels were not significantly different from those of control rats at each corresponding time point. When fructoseinduced hypertension had been established (from $112 \pm 4 \mathrm{mmHg}$ to $141 \pm 3 \mathrm{mmHg}, p<0.05$ ) in rats fed fructose for 4 weeks, subsequent aliskiren treatment for 4 weeks reversed the elevated SBP to the control levels.

Aliskiren does not alter the food intake, water intake, urine flow or body weight gain in fructose-fed rats

Effects of aliskiren on food intake, water intake, urine flow and body weight gain of fructose-fed rats are shown in Figure 2. There were no significant differences in food, water intakes, urine flow or body weight gain among groups at the corres ponding time periods.

\section{Aliskiren prohibits and ameliorates hyperglycaemia, hyperinsulinaemia, hypertriglyceridaemia and hypercholesterolemia in fructose-fed rats}

Table I summarizes the effects of a high fructose diet alone and in combination with aliskiren on plasma glucose, insulin, triglycerides and total cholesterol. Fructose-fed rats significantly increased plasma glucose, insulin, triglyceride and total cholesterol compared to control rats throughout the experiments. Concurrent aliskiren treatment 

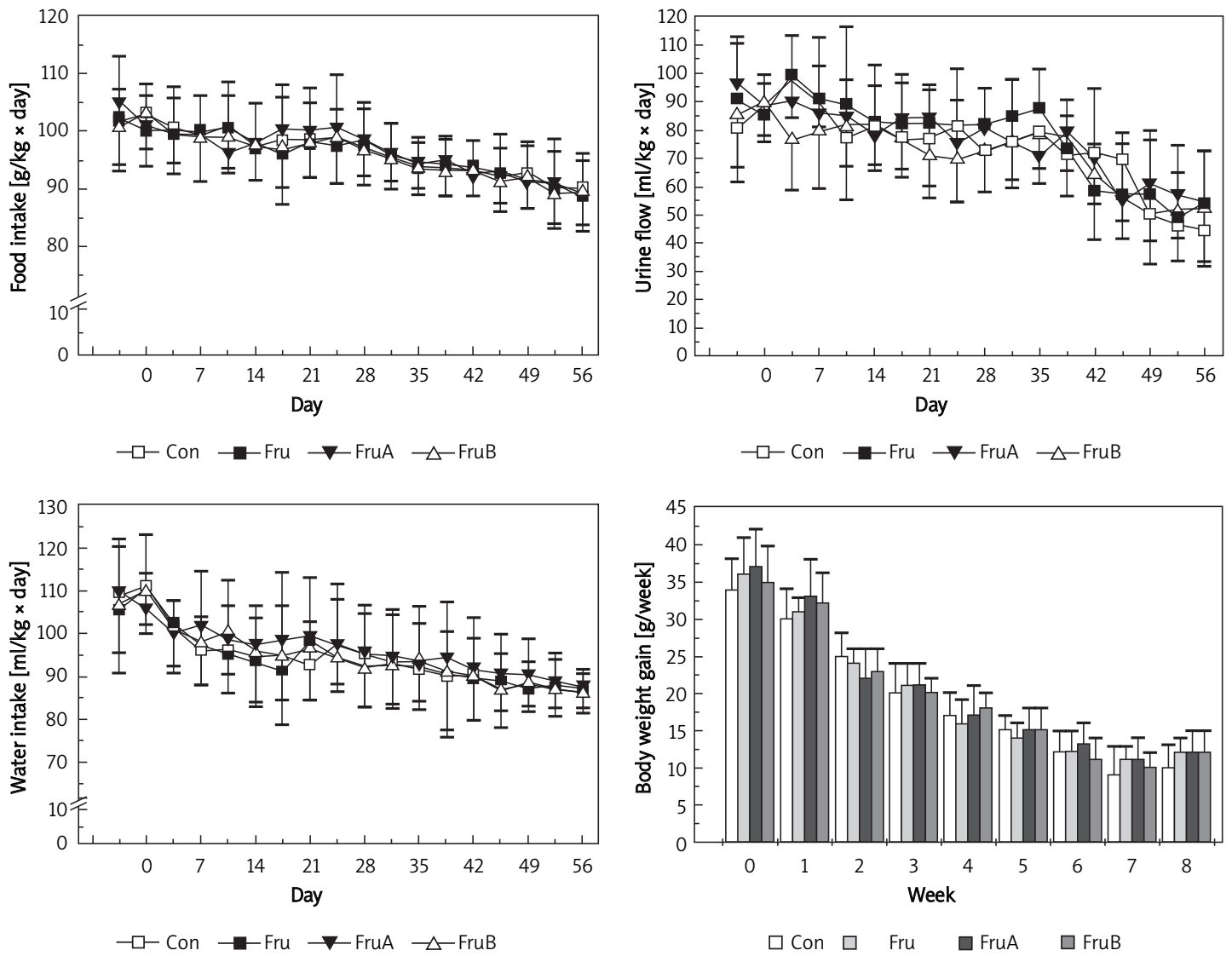

Figure 2. Effects of aliskiren on food intake, water intake, urine flow, and body weight gain of fructose-fed hypertensive rats

Group Con - rats were fed standard chow diet, group Fru - rats were fed high fructose diet, group FruA - rats were fed high fructose diet and were co-infused with aliskiren, group FruB - rats were treated as group Fru, but aliskiren was administered 4 weeks later, $n=6$ for each group

interrupted the rise of plasma glucose, insulin, triglycerides and total cholesterol in fructose-fed rats, and the levels were not significantly different from those of control rats at each corresponding time point. In addition, a high fructose diet for 4 weeks induced hyperglycaemia, hyperinsulinaemia, hypertriglyceridaemia and hypercholesterolemia, and subsequently in combination with aliskiren treatment for 4 weeks reversed these elevated parameters to the control levels.

\section{Aliskiren suppresses and improves insulin resistance in fructose-fed rats}

The effect of aliskiren on insulin resistance of fructose-fed rats is presented in Figure 3. There were no significant differences in HOMA-IR values (between $6.417 \pm 0.99$ and $6.35 \pm 1.25$ ) in control rats throughout the experiments. A high fructose diet significantly increased HOMA-IR values from baseline $(6.15 \pm 1.59)$ to $21.25 \pm 2.08$ and $21.28 \pm 3.1$ $(p<0.05)$ at week 4 and 8 , respectively, and significantly induced metabolic syndrome.

Fructose-fed rats had significantly higher HOMA-IR values at day 28 and 56 (from baseline
$6.15 \pm 1.59$ to $21.25 \pm 2.08$ and $21.28 \pm 3.1$ at day 28 and 56 , respectively, $p<0.05)$ than those of control rats and pre-fructose feeding. The levels of HOMA-IR (between $5.98 \pm 1.14$ and $7.05 \pm 0.93$ ) in fructose-fed rats with concomitant aliskiren treatment were not significantly different from those of control rats at each corresponding time point. Furthermore, when fructose-induced higher HOMA-IR level had been established (from $6.2 \pm 0.67$ to $19.97 \pm 3.93, p<0.05$ ), subsequent treatment with aliskiren for 4 weeks inverted the elevated HOMA-IR to the control levels (from 19.97 \pm 3.93 to $6.37 \pm 0.92, p<0.05)$.

\section{Discussion}

In this rat model of fructose-fed diet, we have demonstrated that a high fructose diet elicits the elevation of plasma glucose, insulin, triglycerides, total cholesterol as well as SBP. These results, consistent with reports by others [12-14], support the view that a sustained fructose-fed diet causes hypertensive insulin resistance in rats. Moreover, we found that administration of aliskiren, a direct renin inhibitor, not only prevented but also reversed 
Table I. Effects of high fructose diet alone and in combination with aliskiren on plasma glucose, insulin, triglycerides and total cholesterol

\begin{tabular}{|c|c|c|c|c|}
\hline Group & $N$ & Day 0 & Day 28 & Day 56 \\
\hline \multicolumn{5}{|c|}{ Glucose [mmol/l] } \\
\hline Con & 6 & $6.45 \pm 0.81$ & $6.33 \pm 0.47$ & $6.90 \pm 0.20$ \\
\hline Fru & 6 & $6.46 \pm 0.79$ & $10.54 \pm 0.89^{\star \#}$ & $11.86 \pm 1.47^{\star \# \#}$ \\
\hline FruA & 6 & $6.42 \pm 0.68$ & $6.49 \pm 0.22$ & $7.07 \pm 0.45$ \\
\hline FruB & 6 & $6.31 \pm 0.45$ & $10.67 \pm 1.40^{* \#}$ & $6.74 \pm 0.41$ \\
\hline \multicolumn{5}{|c|}{ Insulin [pmol/l] } \\
\hline Con & 6 & $161.6 \pm 20.8$ & $155.6 \pm 19.6$ & $148.4 \pm 26.7$ \\
\hline Fru & 6 & $153.1 \pm 30.0$ & $310.0 \pm 32.2^{* \#}$ & $289.6 \pm 22.5^{\text {*\# }}$ \\
\hline FruA & 6 & $150.8 \pm 25.6$ & $149.0 \pm 27.7$ & $161.6 \pm 24.6$ \\
\hline FruB & 6 & $159.2 \pm 20.5$ & $301.6 \pm 36.3^{\star \#}$ & $160.3 \pm 23.0$ \\
\hline \multicolumn{5}{|c|}{ Triglycerides [mmol/l] } \\
\hline Con & 6 & $1.56 \pm 0.09$ & $1.60 \pm 0.10$ & $1.55 \pm 0.08$ \\
\hline Fru & 6 & $1.60 \pm 0.15$ & $3.13 \pm 0.23^{\star \#}$ & $3.34 \pm 0.25^{\text {\#\# }}$ \\
\hline FruA & 6 & $1.55 \pm 0.12$ & $1.59 \pm 0.09$ & $1.72 \pm 0.21$ \\
\hline FruB & 6 & $1.50 \pm 0.09$ & $3.15 \pm 0.17^{\star \#}$ & $1.68 \pm 0.13$ \\
\hline \multicolumn{5}{|c|}{ Total cholesterol [mmol/l] } \\
\hline Con & 6 & $2.21 \pm 0.31$ & $2.27 \pm 0.14$ & $2.20 \pm 0.13$ \\
\hline Fru & 6 & $2.25 \pm 0.17$ & $3.24 \pm 0.14^{\star \#}$ & $3.30 \pm 0.13^{\star \#}$ \\
\hline FruA & 6 & $2.42 \pm 0.15$ & $2.38 \pm 0.23$ & $2.31 \pm 0.15$ \\
\hline FruB & 6 & $2.33 \pm 0.11$ & $3.16 \pm 0.20^{\star \# \#}$ & $2.42 \pm 0.21$ \\
\hline
\end{tabular}

Con indicates control rats with normal chow diet, Fru - fructose-fed diet, FruA - fructose-fed diet with concurrent aliskiren administration, FruB - fructose-fed diet and further aliskiren administration after 4 weeks of fructose-fed diet; ${ }^{*} p<0.05$ vs. day $0,{ }^{*} p<0.05$ vs. control group

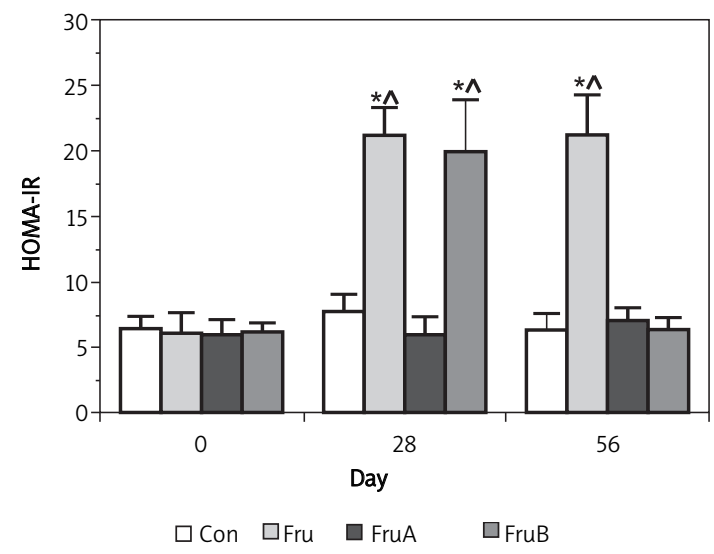

Figure 3. Effect of aliskiren on insulin resistance of fructose-fed rats

Group Con - rats were fed standard chow diet, group Fru rats were fed high fructose diet, group FruA - rats were fed high fructose diet and were co-infused with aliskiren, group FruB - rats were treated as group Fru, but aliskiren was administered 4 weeks later, HOMA-IR - homeostasis model assessment of insulin resistance, ${ }^{*}$ and ${ }^{\wedge}$ denote $p<0.05 \mathrm{vs}$ pre-fructose and control rats, respectively, $n=6$ for each group the development of hypertension, hyperglycaemia, hyperinsulinaemia, hypertriglyceridaemia, hypercholesterolemia and insulin resistance, suggesting that renin-associated mechanisms play a pivotal role in the development of metabolic syndrome in rats fed with a high fructose diet.

Several studies have shown that RAS plays a cardinal role in the pathogenesis of hypertension in fructose-fed rats $[15,16]$. In this present study, we have shown that a direct renin inhibitor can prevent and reverse the rise of systolic blood pressure in fructose-fed rats, and the mechanism may involve suppressing the generation of Ang II. The other studies also showed that elevation of plasma Ang II and tissue Ang II type 1 receptor played a pivotal role in the pathogenesis of fructose-fed hypertensive rats $[15,16]$. Additionally, chronic ACE inhibitor or ARB treatment has been shown to decrease the rise of blood pressure and restores the activity of endothelial nitric oxide synthase in fructose-fed rats $[17,18]$.

Renin is the rate-limiting step in the generation of Ang II, and a direct renin inhibitor suppressed the generation of Ang II. Recently, one clinical study showed that direct renin inhibition, by aliskiren, might be efficacious in reducing blood pressure and improving insulin sensitivity in hypertensive patients with metabolic syndrome [19]. Several experimental studies have also shown that aliskiren might improve insulin sensitivity and protect against pancreatic injury in animal models of obesity, type 2 diabetes or Ren2 rats [20-23]. The mechanisms for the direct renin inhibitor to prevent as well as reverse insulin resistance may act through inhibition of the production of Ang II, and down-regulation of the local RAS in pancreatic islet tissue and adipose tissue. Numerous studies have illustrated that Ang II plays an important role in insulin resistance [24-27]. For example, Ang II acting through Ang II type 1 receptor can inhibit insulin signalling pathways and translocation of glucose transporter 4 in both non-diabetic and diabetic rats, and then facilitates the development of insulin resistance $[24,28]$. In addition, Ang II may induce the development of insulin resistance by its vasoconstrictor actions, thus decreasing blood flow and glucose uptake into insulin-sensitive tissues [25]. Moreover, Ang II infusion induces endothelial dysfunction [26], hepatic insulin resistance and elevations in blood pressure [27].

In the present study, we have demonstrated that a direct renin inhibitor can improve insulin resistance in fructose hypertensive rats. This observation is consistent with the study from Habibi et al., who showed that renin inhibition attenuated insulin resistance, oxidative stress and pancreatic remodelling in the transgenic Ren2 rat [29]. 
Similarly, blockade of the effects of Ang II may improve blood flow to insulin-sensitive tissues and increase islet blood flow for an adequate insulin release [30]. Several animal studies have also demonstrated that inhibition of the RAS with an ACE inhibitor or ARB not only prevents but also reduces hypertension, and improves insulin resistance in a high fructose-fed animal model [25, 31]. Furthermore, interruption of the RAS via chronic ACE inhibition or ARB reduces oxidative stress and restores NO production in fructose hypertensive rats $[15,17,18]$, which can lead to improved insulin signalling and resistance in pancreas and skeletal muscle [7, 30, 32].

Whether or not inhibition of RAS can blunt the rise of triglycerides in fructose-fed hypertensive rats is still under debate. A study [33] reported that either ACE inhibitor or ET blockade did not decrease the hypertriglyceridaemia in fructose hypertensive rats, although the combination of both inhibitors significantly diminished hypertriglyceridaemia in fructose hypertensive rats. However, another study showed that blockade of the RAS via ACE inhibitor or ARB could reduce hypertension and hypertriglyceridaemia and improve insulin sensitivity [34]. In the present study, aliskiren not only prevented but also reversed hypertriglyceridaemia and hypercholesterolemia to normal levels in fructose hypertensive rats. The mechanism of action for aliskiren is unclear and may be due to downregulation of local RAS in adipose and liver tissue. A recent study in obese mice fed with a high fat diet demonstrated that systemic inhibition of renin via aliskiren elicited a decline of adipose Ang I and Ang II, visceral adiposity and plasma leptin. Furthermore, aliskiren normalized adipose tissue function, and allowed triglycerides to be stored in adipocytes and not in other tissues, and improved insulin resistance [35]. Another study showed that $A R B$ reduced hepatic plasminogen activator inhibitor-1 gene expression, decreased hepatic triglyceride accumulation, and improved insulin resistance [36].

The limitations of this study were the small number of animals in each group and the lack of a vehicle-treated group. In addition, the concentrations of local and systemic Ang II and its correlation with the changes in study variables were not measured.

In conclusion, the present study demonstrates that a high fructose diet significantly induces metabolic syndrome (hypertension, hyperglycaemia, insulin resistance, hypertriglyceridaemia and hypercholesterolaemia) and is independent of changes in food and water intake, urine flow and body weight gain. Aliskiren, a direct renin inhibitor, not only prevents but also reverses the development of metabolic syndrome in rats fed with a high fructose diet. In future, aliskiren-related benefits could be expanded in the clinical setting of the metabolic syndrome. Nevertheless, this remains to be further confirmed by clinical studies.

\section{Acknowledgments}

We are thankful to the Taiwan Society of Nephrology for providing 2009 research grants for this study. This study was presented at the 2010 Congress of the European Renal Association European Dialysis and Transplant Association (Munich, Germany, June 25-28, 2010).

\section{References}

1. Reaven GM. Banting lecture 1988. Role of insulin resistance in human disease. Diabetes 1988; 37: 1595-607.

2. Kostapanos MS, Liamis GL, Elisaf M. Features of the metabolic syndrome relating to cardiorenal outcomes. Arch Med Sci 2008; 4: 424-6.

3. Ferder L, Ferder MD, Inserra F. The role of high-fructose corn syrup in metabolic syndrome and hypertension. Curr Hypertens Rep 2010; 12: 105-12.

4. Ferrario CM. Importance of the renin-angiotensin-aldosterone system (RAS) in the physiology and pathology of hypertension. An overview. Drugs 1990; 39 Suppl 2: 1-8.

5. Fang TC, Huang WC. Angiotensin receptor blockade blunts hyperinsulinemia-induced hypertension in rats. Hypertension 1998; 32: 235-42.

6. Fang TC, Huang WC. Role of angiotensin II in hyperinsulinemia-induced hypertension in rats. J Hypertens 1998; 16: 1767-74.

7. Abuissa H, Jones PG, Marso SP, et al. Angiotensinconverting enzyme inhibitors or angiotensin receptor blockers for prevention of type 2 diabetes: a meta-analysis of randomized clinical trials. J Am Coll Cardiol 2005; 46: 821-6.

8. Gillespie EL, White CM, Kardas M, et al. The impact of ACE inhibitors or angiotensin II type 1 receptor blockers on the development of new-onset type 2 diabetes. Diabetes Care 2005; 28: 2261-6.

9. Wood JM, Schnell CR, Cumin F, et al. Aliskiren, a novel, orally effective renin inhibitor, lowers blood pressure in marmosets and spontaneously hypertensive rats. J Hypertens 2005; 23: 417-26.

10. Yarows SA, Oparil S, Patel S, et al. Aliskiren and valsartan in stage 2 hypertension: subgroup analysis of a randomized, double-blind study. Adv Ther 2008; 25: 1288-302.

11. Parving HH, Persson F, Lewis JB, et al. Aliskiren combined with losartan in type 2 diabetes and nephropathy. N Engl J Med 2008; 358: 2433-46.

12. Huang BW, Chiang MT, Yao HT, et al. The effect of highfat and high-fructose diets on glucose tolerance and plasma lipid and leptin levels in rats. Diabetes Obes Metab 2004; 6: 120-6.

13. Thirunavukkarasu V, Anitha Nandhini AT, Anuradha CV. Cardiac lipids and antioxidant status in high fructose rats and the effect of alpha-lipoic acid. Nutr Metab Cardiovasc Dis 2004; 14: 351-7.

14. Reungjui S, Roncal CA, Mu W, et al. Thiazide diuretics exacerbate fructose-induced metabolic syndrome. J Am Soc Nephrol 2007; 18: 2724-31.

15. Shinozaki K, Ayajiki K, Nishio Y, et al. Evidence for a causal role of the renin-angiotensin system in vascular 
dysfunction associated with insulin resistance. Hypertension 2004; 43: 255-62.

16. Iyer SN, Katovich MJ, Raizada MK. Changes in angiotensin AT1 receptor density during hypertension in fructose-fed rats. Adv Exp Med Biol 1996; 396: 49-58.

17. Miatello R, Risler N, Gonzalez S, et al. Effects of enalapril on the vascular wall in an experimental model of syndrome X. Am J Hypertens 2002; 15: 872-8.

18. Miatello R, Risler N, Castro C, et al. Chronic administration of losartan reverses cardiovascular changes in hypertensive fructose-fed rats. Cell Mol Biol (Noisy-legrand) 2003; 49: 945-52

19. Fogari R, Zoppi A, Mugellini A, et al. Different effects of aliskiren and losartan on fibrinolysis and insulin sensitivity in hypertensive patients with metabolic syndrome. Horm Metab Res 2010; 42: 892-6.

20. Kang YS, Lee MH, Song HK, et al. Aliskiren improves insulin resistance and ameliorates diabetic vascular complications in db/db mice. Nephrol Dial Transplant 2011; 26: 1194-204.

21. Iwai $\mathrm{M}$, Kanno H, Tomono $\mathrm{Y}$, et al. Direct renin inhibition improved insulin resistance and adipose tissue dysfunction in type 2 diabetic KK-A(y) mice. J Hypertens 2010; 28: 1471-81

22. Lastra G, Habibi J, Whaley-Connell AT, et al. Direct renin inhibition improves systemic insulin resistance and skeletal muscle glucose transport in a transgenic rodent model of tissue renin overexpression. Endocrinology 2009; 150: 2561-8.

23. Dong YF, Liu L, Kataoka K, et al. Aliskiren prevents cardiovascular complications and pancreatic injury in a mouse model of obesity and type 2 diabetes. Diabetologia 2010; 53: 180-91.

24. Igarashi M, Hirata A, Nozaki H, et al. Role of angiotensin II type- 1 and type- 2 receptors on vascular smooth muscle cell growth and glucose metabolism in diabetic rats. Diabetes Res Clin Pract 2007; 75: 267-77.

25. Iyer SN, Katovich MJ. Effect of acute and chronic losartan treatment on glucose tolerance and insulin sensitivity in fructose-fed rats. Am J Hypertens 1996; 9: 662-8.

26. Diep QN, El Mabrouk M, Cohn JS, et al. Structure, endothelial function, cell growth, and inflammation in blood vessels of angiotensin II-infused rats: role of peroxisome proliferator-activated receptor-gamma. Circulation 2002; 105: 2296-302.

27. Rao RH. Effects of angiotensin II on insulin sensitivity and fasting glucose metabolism in rats. Am J Hypertens 1994; 7: 655-60.

28. Folli F, Saad MJ, Velloso L, et al. Crosstalk between insulin and angiotensin II signalling systems. Exp Clin Endocrinol Diabetes 1999; 107: 133-9.

29. Habibi J, Whaley-Connell A, Hayden MR, et al. Renin inhibition attenuates insulin resistance, oxidative stress, and pancreatic remodeling in the transgenic Ren2 rat. Endocrinology 2008; 149: 5643-53.

30. Carlsson PO, Berne C, Jansson L. Angiotensin II and the endocrine pancreas: effects on islet blood flow and insulin secretion in rats. Diabetologia 1998; 41: 127-33.

31. limura O, Shimamoto K, Matsuda K, et al. Effects of angiotensin receptor antagonist and angiotensin converting enzyme inhibitor on insulin sensitivity in fructose-fed hypertensive rats and essential hypertensives. Am J Hypertens 1995; 8: 353-7.

32. Engeli S, Schling P, Gorzelniak K, et al. The adipose-tissue renin-angiotensin-aldosterone system: role in the metabolic syndrome? Int J Biochem Cell Biol 2003; 35 807-25.
33. Ezra-Nimni O, Ezra D, Peleg E, et al. Trandolapril and endothelin antagonist LU-135252 in the treatment of the fructose-induced hypertensive, hyperinsulinemic, hypertriglyceridemic rat. Am J Hypertens 2003; 16: 324-8.

34. Furuhashi M, Ura N, Takizawa H, et al. Blockade of the renin-angiotensin system decreases adipocyte size with improvement in insulin sensitivity. J Hypertens 2004; 22 1977-82.

35. Stucchi P, Cano V, Ruiz-Gayo M, et al. Aliskiren reduces body-weight gain, adiposity and plasma leptin during dietinduced obesity. Br J Pharmacol 2009; 158: 771-8.

36. Rosselli MS, Burgueno AL, Carabelli J, et al. Losartan reduces liver expression of plasminogen activator inhibitor-1 (PAI-1) in a high fat-induced rat nonalcoholic fatty liver disease model. Atherosclerosis 2009; 206: $119-26$. 